\title{
DISSEMINATED CRYPTOCOCCOSIS SUCCESSFULLY TREATED WITH AMPHOTERICIN B.
}

\author{
Major R. O. WALTERS, M.B., M.R.C.P.(U.K.), D.C.H., D.T.M.\&H., R.A.M.C. \\ British Military Hospital, Rinteln
}

Introduction

DISSEMINATED cryptococcosis is rare in Britain occurring most often as an opportunist infection in association with chronic debilitating disease, malignant disease and in patients receiving prolonged steroid therapy. Wilson et al (1970) suggested that the infection was more common in Britain than generally realised and pointed to the need for physicians and bacteriologists to be alert to the possibility of cryptococcosis and other systemic mycoses. A case of disseminated cryptococcosis, which was diagnosed by skin biopsy and culture of sputum and cerebrospinal fluid (c.s.f.) and successfully treated with amphotericin $B$, is presented.

\section{Case report}

A 42 year old housewife was admitted to the Military Hospital, Colchester on 18 March 1971 with an eleven day history of severe headaches, vomiting and drowsiness. She had a previous history of sarcoidosis in August 1962 characterised by uveitis, parotid swelling and bilateral hilar adenopathy, confirmed by parotid biopsy. She was treated with steroids from November 1962 to February 1964 when she was discharged well. In November 1970, she had developed fatigue, malaise, weight loss and a productive cough with small haemoptyses and had been investigated with negative results. She had lived all her life in south east England, had no history of direct contact with pigeons but had kept budgerigars as household pets for 14 years. On examination the only abnormal physical findings were a persistent low grade pyrexia, tenderness under the right costal margin, slight neck stiffness and a small blue-tinged subcutaneous nodule on the inner aspect of the left thigh. Investigation revealed an E.S.R. (Westergren) of $10-25 \mathrm{~mm} / \mathrm{hr}$, white cell count, liver function tests, plasma proteins, serum calcium and plasma cortisol levels being normal. Chest X-rays showed patchy shadowing in both mid zones. Several examinations of c.s.f. showed high protein and low glucose levels and an increased cell count (Table I); no bacteria or fungi were identified on direct microscopy, and cultures on blood agar and Lowenstein-Jensen slopes were sterile. Heaf test was negative.

Tuberculosis was strongly suspected and treatment was started with streptomycin, para-aminosalicylic acid, isoniazid and pyridoxine. The development of focal neurological signs, which proved to be transient, led to her transfer to a neuro-surgical centre where e.e.g., brain scan and right carotid angiogram failed to show localising features. After initial improvement on anti-tuberculosis chemotherapy she was discharged home on 4 May 1971, but headaches, vertigo and vomiting accompanied by persistent low grade pyrexia, slight neck stiffness and ataxia recurred in July 1971 and she was readmitted. E.S.R. was $14 \mathrm{~mm} / \mathrm{hr}$, c.s.f. showed high protein and low glucose levels and an increased cell count, no bacteria or fungi were seen and culture was sterile. Caloric tests and audiometry showed greatly diminished labyrinthine function. It was felt that 
Table I

Cerebrospinal fluid findings

\begin{tabular}{|c|c|c|c|c|c|}
\hline Date & $\underset{(\mathrm{mg} / 100 \mathrm{ml})}{\text { Protein }}$ & $\begin{array}{c}\text { Glucose } \\
(\mathrm{mg} / 100 \mathrm{ml})\end{array}$ & $\underset{\text { (per ul) }}{\mathrm{RBC}}$ & $\begin{array}{c}\text { WBC } \\
\text { (per ul) }\end{array}$ & $\begin{array}{l}\text { Polymorphs } \\
\text { (per cent) }\end{array}$ \\
\hline 19. 3.71 & 172 & 62 & 100 & 90 & 46 \\
\hline 20. 3.71 & 140 & 75 & 30 & 150 & 40 \\
\hline 23. 3.71 & 200 & 30 & 40 & 100 & 43 \\
\hline 2. 4.71 & 220 & 32 & 2 & 6 & 0 \\
\hline 5. 7.71 & 200 & 16 & 0 & 325 & 19 \\
\hline 12. 7.71 & 200 & 5 & 50 & 20 & 0 \\
\hline 11. 5.72 & 400 & - & 13 & 0 & 0 \\
\hline 6. 7.72 & 250 & 18 & 37 & 46 & 10 \\
\hline 28. 7.72 & 230 & 20 & - & - & - \\
\hline 21.9 .72 & .76 & 35 & 0 & 0 & 0 \\
\hline 11.10 .72 & 73 & 35 & 2 & 0 & 0 \\
\hline 7.11 .72 & 130 & - & 130 & 2 & 0 \\
\hline 7.12 .72 & 70 & 42 & 0 & 0 & 0 \\
\hline 18. 1.73 & 75 & 45 & 62 & 0 & 0 \\
\hline 13. 4.73 & 30 & 65 & 0 & 0 & 0 \\
\hline
\end{tabular}

Note: Amphotericin $B$ was commenced on 27.7 .72 and completed on 8.10.72.

her vertigo and ataxia were due to labyrinthine damage caused by streptomycin which was discontinued. Sarcoidosis was considered as a possible diagnosis and a Kveim test was performed; the biopsy showed epithelioid granulomata, typical of a positive result which it was felt confirmed the diagnosis. Symptomatic improvement took place on continued treatment with isoniazid and pyridoxine and she was discharged home on 1 September 1971. She remained reasonably well, but in March 1972 she seemed less well with headaches and vomiting again becoming more common. On examination, she had an indolent ulcer on the inner aspect of the left thigh at the site of the nodule noted on her first admission. Excision biopsy of the ulcer was performed on 9 May 1972; microscopy showed a chronic cutaneous granulomatous inflammation with multinucleated giant cells containing clusters of discoid organisms some of which were budding; the organisms were PAS, Gomori silver methenamine and mucicarmine positive and the appearances were considered to indicate Cryptococcus neoformans infection (Figs 1 to 4 ).

In view of this, review of the diagnosis of sarcoidosis was undertaken. Cerebrospinal fluid showed high protein and low sugar levels and an increased cell count; indian ink preparations were negative; cultures on Sabouraud agar at $22^{\circ}$ and $37^{\circ} \mathrm{C}$ yielded yeast colonies having the morphological, cultural and physiological properties of Cryptococcus neoformans sensitive to amphotericin B $0.1 \mu \mathrm{g} / \mathrm{ml}$ and 5-fluorocytosine. 


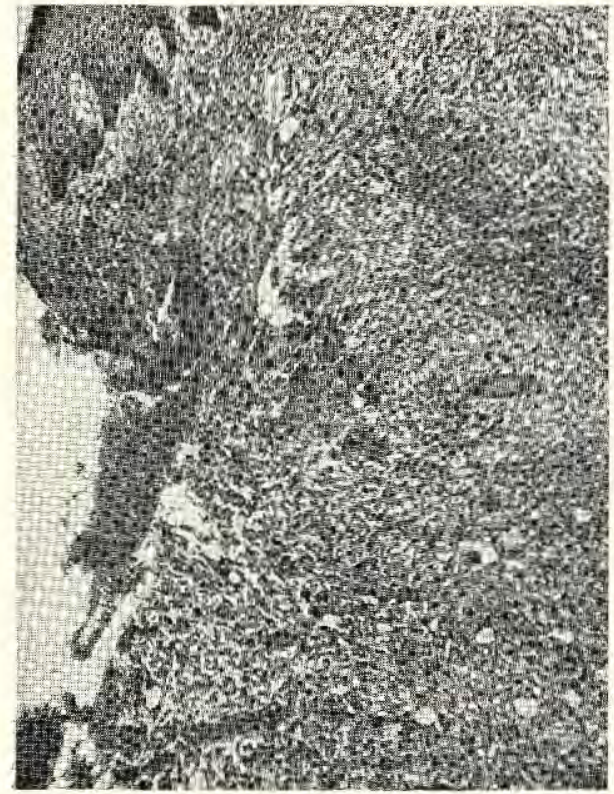

Fig. 1. Skin biopsy $\times 250$ showing mixed acute and chronic granulomatous inflamatory reacho $\perp$ in the dermis.

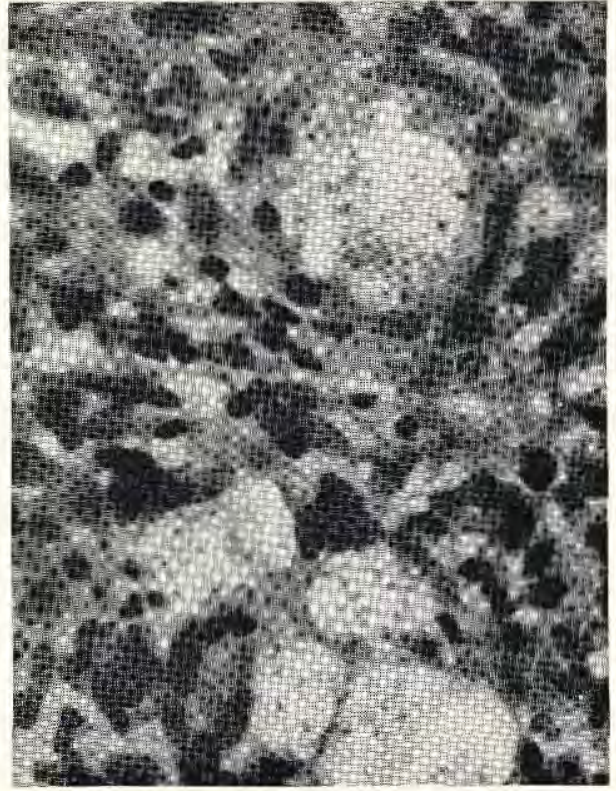

Fig. 2. Skin biopsy $\times 250$ showing numcrous cryptococci in macrophages.

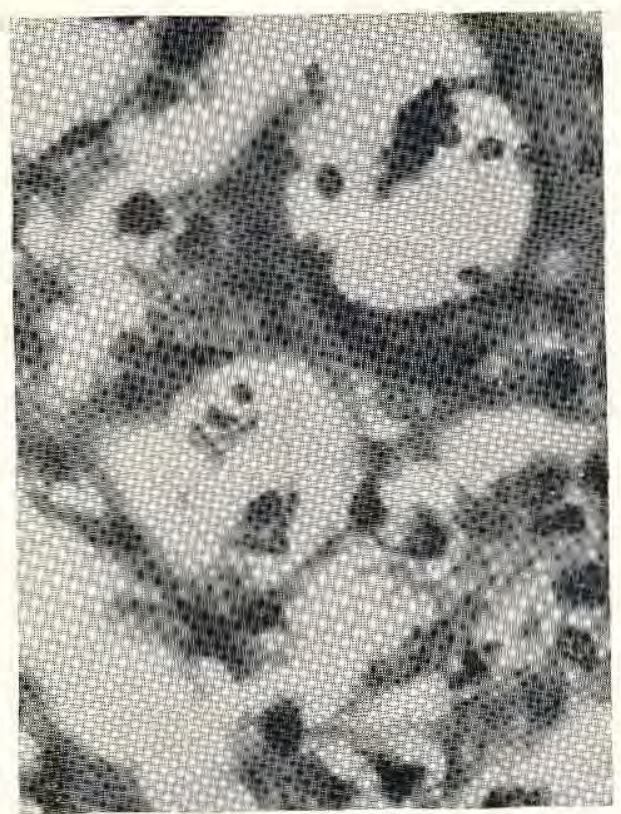

Fig. 4. Skin biopsy $x 400$ PAS showing numerous organisms in the cytoplasm of macrophages and giant cells. 
Cryptococcus neoformans was also cultured from one specimen of sputum. Blood and urine cultures were sterile. Serological tests for cryptococci on serum and c.s.f. were negative, as was the histoplasmin skin test. A diagnosis of disseminated cryptococcosis was made and treatment with intravenous amphotericin $\mathbf{B}$ and a low thiamine diet started on 27 July 1972. The drug was administered by the method described by HildickSmith et al (1964), potassium supplements being given orally. The initial dose of $10 \mathrm{mg}$ was increased to $50 \mathrm{mg}$ on the third day this dose giving minimum serum amphotericin $B$ levels in the range of $0.012-0.16 \mu \mathrm{g} / \mathrm{ml}$. Treatment was complicated by anaemia due to marrow suppression and renal impairment both of which responded to temporary cessation of treatment. Fever, malaise and nausea also occurred, but when a total dose of $2.33 \mathrm{~g}$ amphotericin $\mathrm{B}$ had been given the severity of the drug reaction became sufficient to prevent further treatment. Since the end of treatment she had felt very well and she has had no recurrence of symptoms although she remains ataxic; her c.s.f. returned to normal over a period of six months, no Cryptococcus neoformans being isolated; the pulmonary opacities seen on chest X-ray have slowly resolved.

\section{Discussion}

Cryptococcus neoformans is a true yeast that has a world-wide distribution in soil; there have been many reports of its isolation from pigeon excreta (Partridge and Winner 1966) and of the suitability of bird droppings including those of canaries as a culture medium for Cryptococcus neoformans (Symmers 1967). The portal of entry is believed to be the respiratory tract and infection may involve the central nervous system, lungs, skin, kidneys and bones. Cryptococcal meningitis is the most frequent clinical picture, pulmonary involvement may be asymptomatic or associated with fever, cough and haemoptysis, and cutaneous lesions occur in about one sixth of cases with disseminated disease. It seems probable that in this patient pulmonary infection occurred first, dissemination to the meninges and skin following about 12 months later, but the source of infection remains uncertain although budgerigars kept as pets may have been significant.

Cryptococcus neoformans most often causes infection in patients with diseases associated with disturbances of their immune response; it is difficult to relate the occurrence of cryptococcosis in this patient to a benign form of sarcoidosis treated with steroids 6 to 8 years previously, but tuberculin anergy, negative histoplasmin skin test and negative serological tests suggest that her cell mediated immune mechanisms were disturbed although serum immunoglobulins were normal. The positive Kveim test which was found in this patient led to the acceptance of an incorrect diagnosis of sarcoidosis and would appear to be another example of the doubtful specificity of the test; the alternative being that dual pathology was present.

The laboratory diagnosis of cryptococcal meningitis usually depends on the microscopical examination of indian ink preparations of centrifuged c.s.f. in which the findings resemble those found in tuberculosis meningitis. Microscopy for cryptococci is only positive in 50 to 60 per cent of cases (Butler et al 1964, Gordon and Vedder 1966) and further confusion may arise because the yeast cells closely resemble erythrocytes. In this case the method was unreliable, no yeast cells being found on direct microscopy of 7 samples of c.s.f. from 19 March 1971. The diagnosis was finally made by the recognition of cryptococci in a biopsy of a skin lesion resembling a sarcoid nodule which had 
been noted at the initial examination. Culture of Cryptococcus neoformans from sputum and c.s.f. provided confirmation of disseminated infection.

Serological methods are available to aid in the diagnosis of cryptococcosis and it has been suggested that they can be of value in any case of obscure meningitis (Watkins et al 1969). Negative results of both serum and c.s.f. were obtained in this case.

Before the introduction of amphotericin B, disseminated cryptococcosis carried a considerable mortality. The total dose required to treat the disease is not certain; Sarkany (1968) states that at least $1 \mathrm{~g}$ of the drug and one month of therapy is the minimum schedule and Andriole and Kravetz (1962) recommend a total dose of $3 \mathrm{~g}$. In this case a dose of $2.33 \mathrm{~g}$ of amphotericin B was considered adequate and it was decided not to continue treatment with 5-fluorocytosine, which has been used in the successful treatment of cryptococcal meningitis (Watkins et al 1969), because this would have involved the use of another nephrotoxic agent. Success of therapy can be judged by the clinical response and by the inability to culture the fungus from the c.s.f. The side effects of amphotericin B are well known and although they proved problematical in this case they did not prevent the completion of an adequate course of therapy. The use of a low thiamine diet was in accordance with the recommendations of Littman (1962) who stressed the importance of thiamine for the capsule synthesis of Cryptococcus neoformans.

The following points seem worth making: Firstly, disseminated cryptococcosis may present in a chronic form several years after apparent recovery from sarcoidosis; secondly, in cases of obscure chronic pulmonary or meningeal inflammation, cultures of sputum of c.s.f. on Sabouraud agar at $22^{\circ} \mathrm{C}$ and $37^{\circ} \mathrm{C}$ may prove to be diagnostic.

\section{Acknowledgements}

I would like to thank Colonel H. S. Moore late R.A.M.C. and Colonel H. Foster, late R.A.M.C. for permission to publish this case. The diagnosis of cutaneous cryptococcosis was made by the Army Histopathology Reference Laboratory who also produced the illustrations. Assistance was also received from the Mycology Reference Laboratory at the London School of Tropical Medicine and Hygiene.

\section{REFERENCES}

ANDriole, V. T. and Kravetz, H. M. (1962). J. Amer. med. Ass. 180, 269.

Butler, W. T. Alling, D. W. Spickard, A. and Utz, J. P. (1964). New Engl. J. Med. 270, 69.

Gordon, M. A. and Vedder, D. K. (1966). J. Amer. med. Ass. 197, 961.

Hildick-Smith, G., Blank, H. and Sarkany, I. (1964). In. Fungus Disease and Their Treatment. J. \& A. Churchill. London.

LrtTman, M. L. (1962). In. Fungus and Fungus Disease. Charles C. Thomas, Illinois. Chapter 20.

Partridge, B. M. and Winner, H. I. (1966). Lancet ii, 1251.

SARKANY, I. (1968). Recent Advances in Medictine. 15th ed. J. \& A. Churchill. London. Chapter 8.

SYMMERS, W. St. C. (1967). Lancet i, 159-160.

Watkins, J. S., Campbell, M. J., Gardner-Medwin, D., Ingham, H. R. and MurRay, I. G. (1969). Brit. med. J. iii, 29-31.

Wilson, T. S., Fleming, W. A., Robinson, F. L. J. and Nicholl, B. (1970). J. clin. Path. 23, 657-663. 